\title{
Selective localised modifications of silicon crystal by ultrafast laser induced micro-explosion
}

\author{
Ludovic Rapp ${ }^{1}$, Bianca Haberl ${ }^{2}$, Jodie E. Bradby ${ }^{2}$, Eugene G. Gamaly ${ }^{1}$, Jim S. Williams ${ }^{2}$, Saulius Juodkasis ${ }^{3}$ \\ and Andrei V. Rode ${ }^{1}$ \\ ${ }^{1}$ Laser Physics Centre, Research School of Physics and Engineering, The Australian National University, \\ Canberra ACT 0200 Australia \\ ${ }^{2}$ Electronic Materials Engineering, Research School of Physics and Engineering, The Australian National \\ University, Canberra ACT 0200 Australia \\ ${ }^{3}$ Swinburne University of Technology, Hawthorn, VIC, 3122, Australia
}

\begin{abstract}
Femtosecond (fs) laser pulses focused and confined inside the bulk of a material can deposit a volume energy density up to several $\mathrm{MJ} / \mathrm{cm}^{3}$ in a sub-micron volume. This creates highly non-equilibrium, hot, dense and short-lived plasmas with conditions favorable for arrangement of atoms into unusual material phases. Singlecrystal silicon was exposed to strong shock waves induced by laser micro-explosion in confined geometry. The conditions of confinement were realized by focusing $170-\mathrm{fs}$ pulses, with the energy up to $2.5 \mu \mathrm{J}$, on a Si surface buried under a $10-\mu \mathrm{m}$ thick $\mathrm{SiO}_{2}$-layer formed by oxidation of a Si-wafer. The generated intensity was $10^{15} \mathrm{~W} / \mathrm{cm}^{2}$, well above the threshold for optical breakdown and plasma formation. The shock wave modified areas of the Si crystal were sectioned using a focused-ion beam and characterized with scanning electron microscopy. A void surrounded by a shock-wave-modified $\mathrm{Si}$ was observed at the $\mathrm{Si} / \mathrm{SiO}_{2}$ boundary. The results demonstrate that confined micro-explosion opens up new perspectives for studies of high-pressure materials at the laboratory table-top expanding the laser-induced micro-explosion capabilities into the domain of non-transparent materials.
\end{abstract}

Keywords: femtosecond, laser, micro-explosion; silicon, ultrafast, high-pressure

\section{INTRODUCTION}

Under extreme pressure and temperature, as exists inside planets and stars, common materials form new dense phases with unusual physical properties due to compacted atomic arrangement. High-pressure research has revolutionized our understanding of the mineralogy of the deep Earth and other planets. The synthesis and study of new phases of matter at pressures above $100 \mathrm{GPa}(1 \mathrm{Mbar})$ and temperatures above $10^{4} \mathrm{~K}$ (Warm Dense Matter - WDM) may reveal the functional geo-systems of planet and star interiors, as well as for creating materials with extraordinary properties. Many phases that have been predicted theoretically may be realized once appropriate formation conditions are found.

New strategies for materials fabrication are of fundamental importance in the advancement of science and technology. The spectacular developments in high-pressure high-temperature material synthesis, crystal growth, and phaseequilibrium studies were produced in static conditions with diamond-anvil cells (DAC), and in dynamic conditions of shock-wave compression experiments. Pressure and temperature records have increased continuously during the last century, and now it is possible to obtain multimegabar static pressures with simultaneous heating by laser up to $3000 \mathrm{~K}^{1 \text {, }}$ ${ }^{2}$. Even more extreme but transient high pressure/temperature conditions have been created by dynamic methods using explosives and megajoule-class lasers ${ }^{3-6}$.

Both static compression/heating and dynamic shock-wave compression methods involve large multi-Ton expensive equipment, they have very low repeatability rate and yield from nanograms to micrograms of material within volumes of $10^{-6}$ to $10^{-9} \mathrm{~cm}^{3}$. A focus now is on creating new and inexpensive ways of generating reproducible, high-repetition-rate shock waves, with a convincing demonstration of a shock compression utility. 
Confined micro-explosion consists to tightly focalize femtosecond-laser pulses inside a solid material. This technique has several distinctive features making this approach substantially different and attractive for those studies. All phenomena occur on a very short time scale, that allow intensities exceeded $10^{14} \mathrm{~W} / \mathrm{cm}^{2}$ to be reached, resulting in a highly nonlinear light-matter interaction with most dielectrics being ionized early in the laser pulse. Tight focusing a $\sim 100$-fs laser pulse with $100 \mathrm{~nJ}$ energy deep inside a crystal, deposits an energy density of several $\mathrm{MJ} / \mathrm{cm}^{3}$ into a submicron volume, several times higher than the strength (Young modulus) of any material. In such conditions, pressure of the order of millions of atmospheres and temperature in excess are formed by optical breakdown. This causes the solid to superheat and form a plasma with temperature $\sim 1 \mathrm{eV}-10 \mathrm{eV}(10,000 \mathrm{~K}-100,000 \mathrm{~K})$, but with solid-state ion density $\sim 10^{22} \mathrm{~cm}^{-3}-10^{24} \mathrm{~cm}^{-3}$. The confined plasma explodes and generates a powerful shock wave that expands out of the focal volume and compresses the surrounding pristine material. The shock wave of the expanding plasma can reach pressures of over $1 \mathrm{TPa}$ (10 Megabar), triggering dramatic changes in the material properties. New materials with altered physical and chemical properties are formed from the solid-density plasma. Fast quenching of the new phases in micron-size shock-wave affected regions facilitate 'freezing' the non-equilibrium material phases inside the material for further investigation and analysis using x-ray and electron diffraction techniques. The modified material remained confined in a strongly localised region inside a bulk of pristine crystal. This technique opens new strategies for synthesis of new materials into unexplored area of TeraPascal pressure ${ }^{7-9}$.

Recent studies into laser induced confined micro-explosions led to the discovery of a new stable super-dense phase of body-centered-cubic aluminium (bcc-Al) ${ }^{8,9}$, predicted by first-principle theories to exist at pressures higher than 380 $\mathrm{GPa}^{10-13}$, but never before been observed in nature or in laboratory experiments. The new super-dense Al phase was synthesized under non-equilibrium conditions inside sapphire $\left(\alpha-\mathrm{Al}_{2} \mathrm{O}_{3}\right)$. The phase was preserved inside the amorphous compressed sapphire produced from a hot, dense, non-equilibrium, and short-lived plasma. This discovery has demonstrated that ultrafast tightly focused laser pulses can create the unique conditions of extreme pressure and temperature for the formation of high-pressure material phases.

\section{SILICON}

Silicon has a large impact on the world economy because of its wide use in integrated circuits, solar cells, etc... The metastable high-pressure phases of silicon have attracted much attention since they can be easily integrated into existing Si technologies in which their alternative properties can be utilized. At pressures of up to 250GPa (the maximum available in a diamond anvil cell) Si demonstrates 12 different polymorphs with different electronic and optical properties. Diamond-cubic silicon can transform into several different phases under the application of pressure and some phases are stable at room temperature and pressure. It has been shown that during indentation, the diamond cubic phase, $\mathrm{Si}-\mathrm{I}$, transforms to the metallic $\beta$-Sn phase, Si-II. This phase is not stable at ambient pressures. If the pressure is released slowly, Si-II transforms into a mixture of the polycrystalline high-pressure phases Si-XII ${ }^{14-16}$, and Si-III. A fast pressure release is likely to result in a transformation to amorphous silicon (a-Si) (S8). A hexagonal diamond phase, Si-IV, has been reported after heating Si-III above $450 \mathrm{~K}^{17,18}$. There is also a contradiction that currently exists in the literature concerning pressure-induced transformation pathways of both crystalline and amorphous silicon from diamond-anvil and indentation experiments. On loading, Si-I transforms to a metallic $\beta$-Sn structure (Si-II) which undergoes further transformations on unloading to form either a mixture of the phases Si-III (BC8) and Si-XII (r8) or an amorphous silicon $(\alpha-\mathrm{Si})$ structure (so-called pressure-induced $\alpha$-Si). Raman spectroscopy has provided an indispensable and sensitive tool for characterizing these phases and their transformations. Some phases (such as Si-III and Si-XII) are metastable at ambient pressures and temperatures and these can be identified from their unique Raman spectrum ${ }^{19}$.

The potential discovery of new Si phases in the unexplored pressure range of $1000 \mathrm{GPa}$ and above are important from the fundamental point of view of giving further insights into the phase-diagram of silicon. These phases may provide a means for localized band-gap engineering at the nanoscale, in a similar manner to the potential of Si-XII for photovoltaic applications. The ability of ultrafast lasers to locally modify the electronic properties of silicon with submicron precision with repetition rates up to and above $10^{5}$ shots per second opens up opportunities for nanoelectronics through the incorporation of such nanostructures into semiconductor devices. 


\section{LASER-INDUCED MICRO-EXPLOSION}

The experiments were conducted with $170 \mathrm{fs}, 790 \mathrm{~nm}$, laser pulses from a MXR-2001 CLARK laser system. Pulses with up to $2.5 \mu \mathrm{J}$ per pulse were focused using an optical microscope (Olympus IX70) equipped with an oil-immersion $\times 150$ objective $(\mathrm{NA}=1.45)$ The focal spots were measured using a knife-edge technique with a sharp edge of a Si [100] wafer etched at $54.74^{\circ}$ to the surface along the [111] direction and mounted on a nano-positioning stage. The focal spots were measured to have a radius of $0.368 \mu \mathrm{m}$ at the full-width at half-maximum level. The experiments were conducted with laser pulses at $1 \mathrm{kHz}$ repetition rate in a sample moved at a rate $2 \mathrm{~mm} \cdot \mathrm{s}^{-1}$ to guarantee a single shot per spot regime, so that each of the shots was located $2 \mu \mathrm{m}$ apart.

Silicon is not transparent for $790 \mathrm{~nm}$, the wavelength that we used for the experiments. While the laser induced microexplosion method was previously considered as suitable only for transparent materials, we propose to expand it into the unexplored domain of non-transparent materials. To confine laser-matter interaction inside a material the distance ought to be far enough from the crystal surface in order the interaction region can be considered as confined. The interaction of intense laser radiation with matter when the beam is tightly focused inside a transparent material is radically different from the case of focusing the beam onto a surface. In the laser-surface interaction the temperature has maximum at the outermost atomic surface layer. If the absorbed energy density in the surface layer is in excess of ablation threshold, the atomic bonding breaks and the ablated atoms leave the surface. In tightly focused interaction mode the focal zone with high energy density is confined inside a bulk of a cold and dense solid. The laser-affected material remains at the focal area inside the pristine material.

By growing a transparent oxide on the sample, laser induced confined micro-explosion can be applied to virtually any opaque material. The confinement conditions were formed in silicon wafers covered by $10-\mu \mathrm{m}$ thick layer of dioxide of silicon $\left(\mathrm{SiO}_{2}\right) \cdot \mathrm{SiO}_{2}$ is transparent to the laser irradiation and allow tightly focusing femtosecond laser pulses on the buried surface of the $\mathrm{Si}$ crystal. The thickness of the $\mathrm{SiO}_{2}$ layer was not so deep for developing large spherical aberrations with high-NA focusing optics and at the same time guarantee the absence of optical breakdown at the surface.

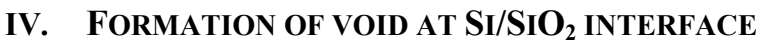

Silicon crystal was exposed to strong shock wave induced by fs-laser micro-explosion in confined geometry ${ }^{20}$. Figure 1 shows a schematic representation of the process and the realization of array of voids using focusing fs-pulses at the $\mathrm{Si} / \mathrm{SiO}_{2}$ interface.

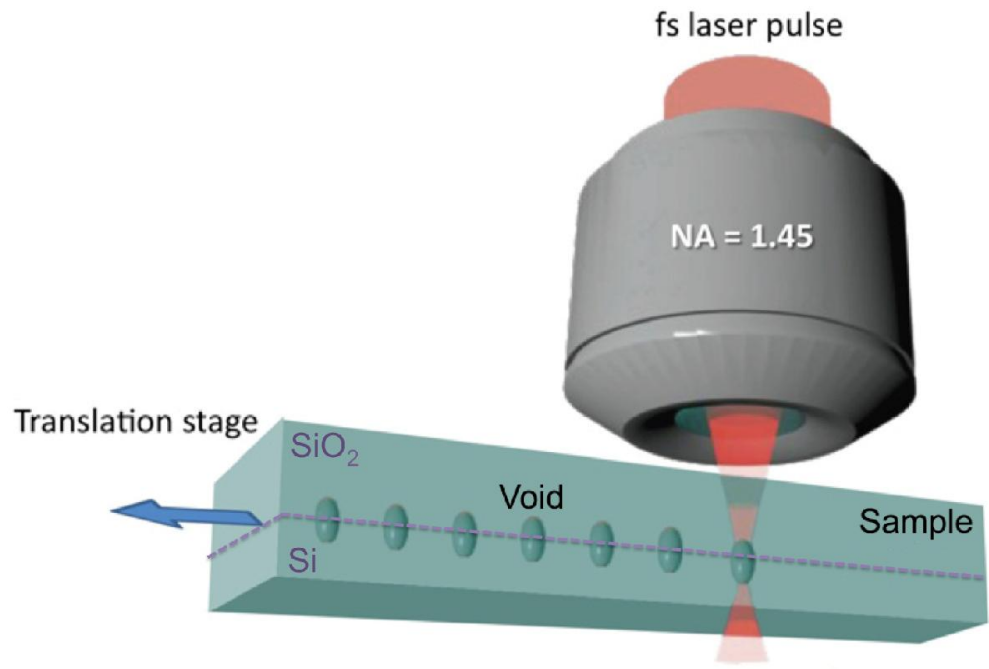

Figure 1. Schematic representation of the laser induced micro-explosion technique at the $\mathrm{Si} / \mathrm{SiO}_{2}$ interface. The dashed line represents the boundary between $\mathrm{Si}$ and $\mathrm{SiO}_{2}$. The elongated spheres represent the voids. 
After processing the array have been investigated by optical microscopy. Figure 2 presents an optical microscope image of a top-view of the sample of the laser-affected areas at the $\mathrm{Si} / \mathrm{SiO}_{2}$ interface. The dark dots in the picture are the voids. The voids were spaced from $2 \mu \mathrm{m}$. The voids are observed through the $\mathrm{SiO}_{2}$ layer.

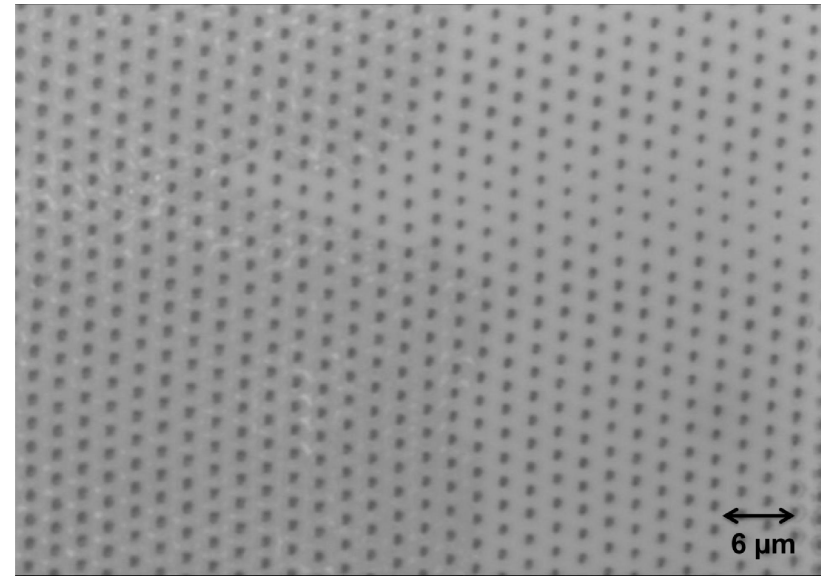

Figure 2. Optical microscope image of arrays of void made at $\mathrm{Si} / \mathrm{SiO}_{2}$ interface viewed through the $\mathrm{SiO}_{2}$ layer produced fs-pulse laser micro-explosion. The spacing between the void is $2 \mu \mathrm{m}$.

To analyze the dimensions of the voids and of the shock wave modified areas, we opened the sample using a focused

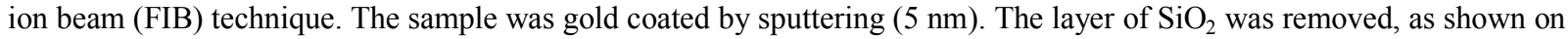
Figure 3a, and then the sample was characterized with scanning electron microscopy (SEM). Figure 3b presents a top-view SEM image of the sample where the $10 \mu \mathrm{m}$ thick $\mathrm{SiO}_{2}$ was removed. In the hole, we can see the Si surface and arrays of voids. Voids were observed under the surface in the region where the fs-laser was focused. Figure $3 \mathrm{c}$ shows the Si surface and the arrays of voids; on the side-view we can observe the $\mathrm{Si} / \mathrm{SiO}_{2}$ boundary.

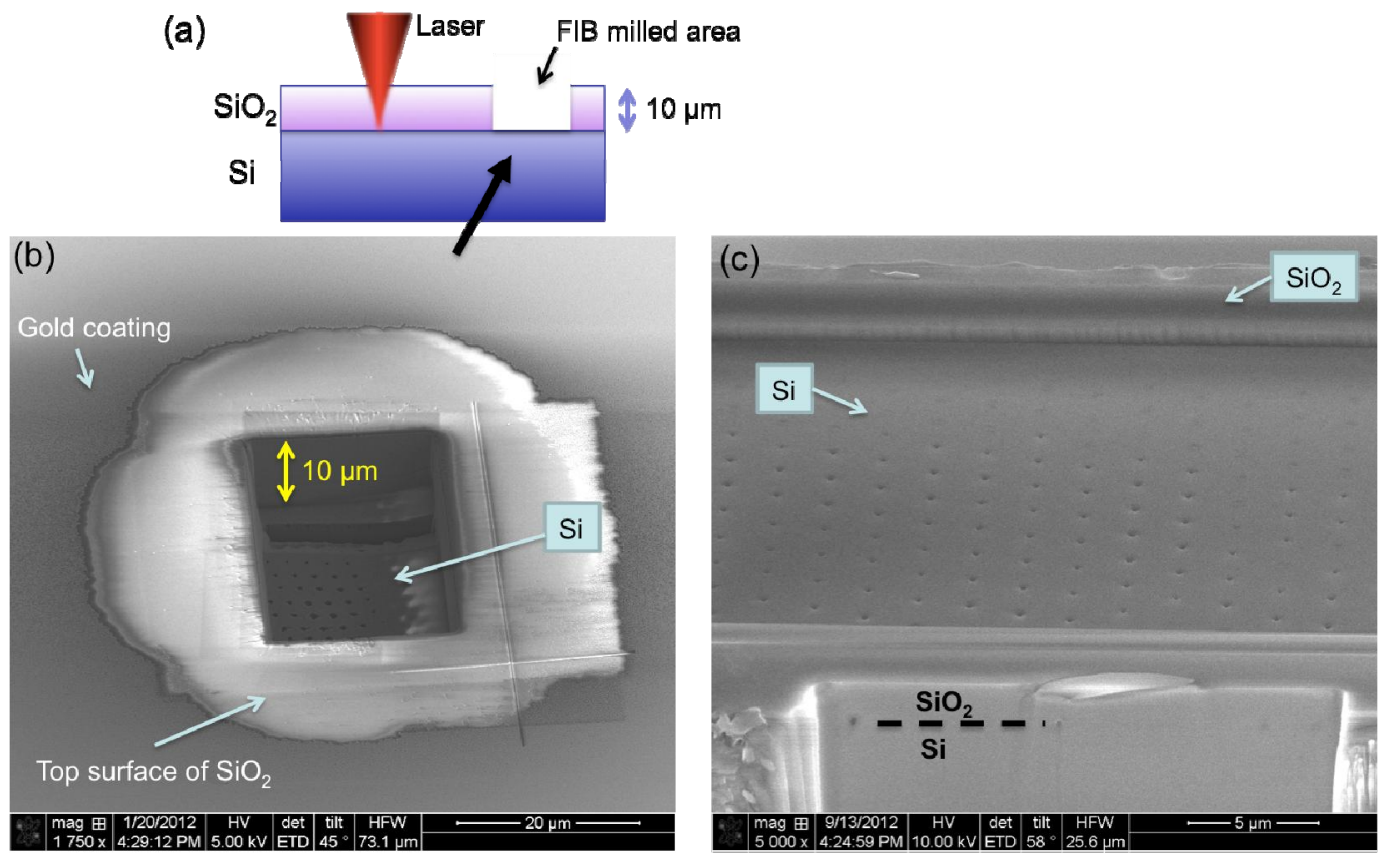

Figure 3. (a) Side-view schematic representation of the processed sample and of the area milled using the focused ion beam (FIB). (b) SEM image of the hole made in the $\mathrm{SiO}_{2}$ layer by FIB to reach the surface of the Si layer, following the schematic in (a). (c) SEM image of the side-view of the milling by $\mathrm{FIB}$ of the $\mathrm{SiO}_{2}$ layer to reach the surface of the $\mathrm{Si}$ layer. 
Each of the regions irradiated by a single laser pulse at the fluence above $\sim 1 \mathrm{~J} / \mathrm{cm}^{2}$ contains a void located at the focal spot. Figure 4 presents arrays of voids at the Si surface produced by $300 \mathrm{~nJ}$ single laser pulses focused $2 \mu \mathrm{m}$ apart with $\times 150$ objective. On the top-view, all the voids had a circular shape and the diameter was $250 \mathrm{~nm}$
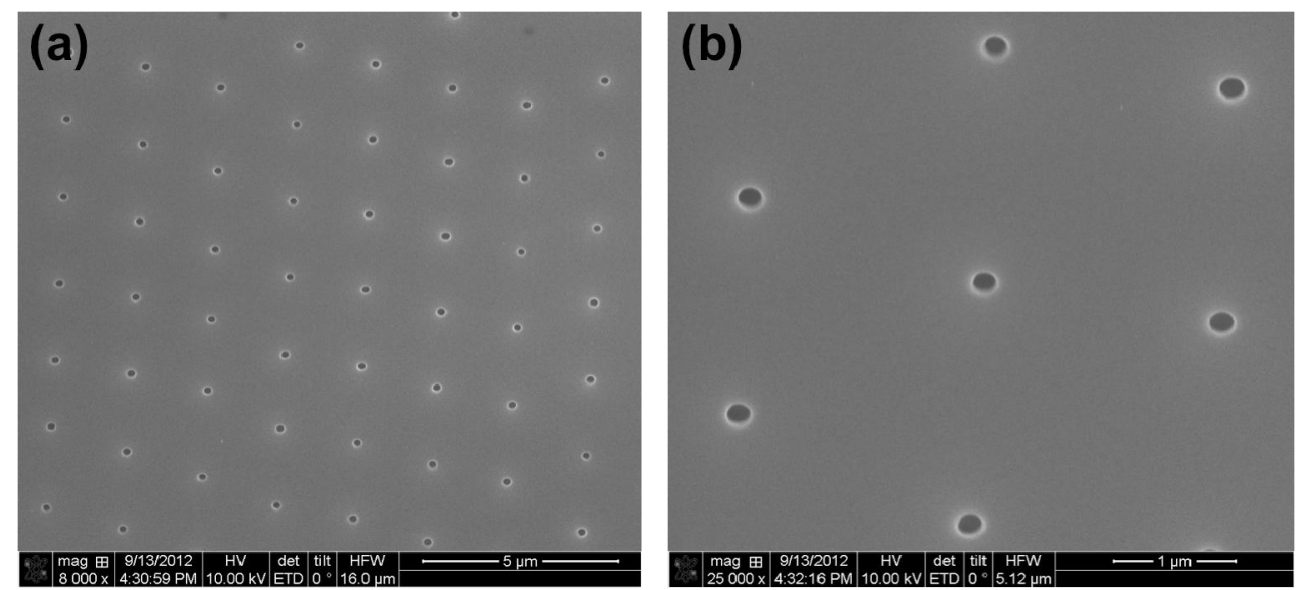

Figure 4. Laser produced voids on a Si-surface buried $10 \mu \mathrm{m}$ beneath the $\mathrm{SiO}_{2}$ surface of the oxidised wafer, the $\mathrm{SiO}_{2}$ was removed using FIB milling, the spacing between the voids is $2 \mu \mathrm{m}$ : (a) SEM image of FIB-opened section showing an array of voids produced by 170 -fs, $800 \mathrm{~nm}, 300 \mathrm{~nJ}$ single laser pulses focused $2 \mu \mathrm{m}$ apart with $\times 150$ objective; (b) Close-view of (a).

On a area where the $\mathrm{SiO}_{2}$ layer was not completely removed, cross-section have been performed using FIB through a void and characterized with SEM. Figure 5 shows a side-view of a void produced with an energy of $700 \mathrm{~nJ}$. The maximal horizontal length of the void in the $\mathrm{SiO}_{2}$ region is $720 \mathrm{~nm}$, in $\mathrm{Si} 550 \mathrm{~nm}$. The vertical size (including $\mathrm{Si}_{\text {and }} \mathrm{SiO}_{2}$ ) is 1.25 $\mu \mathrm{m}$. A shock-wave-modified Si surrounded the voids. The thickness of the boundary between the transparent oxidised layer and crystalline Si where the laser radiation is focused is of the order of only $2 \mathrm{~nm}$, it can be clearly seen in electron microscope. Material should be removed from the energy deposition region in order to form a void involving that a denser shell surrounded the void. Therefore the observation of a large void is unequivocal evidence of creation the pressure well in excess the Young modulus of both materials, $Y_{\mathrm{SiO}_{2}} \sim 75 \mathrm{GPa}$ for $\mathrm{SiO}_{2}$ and $Y_{\mathrm{Si}} \sim 165 \mathrm{GPa}$ for Si.

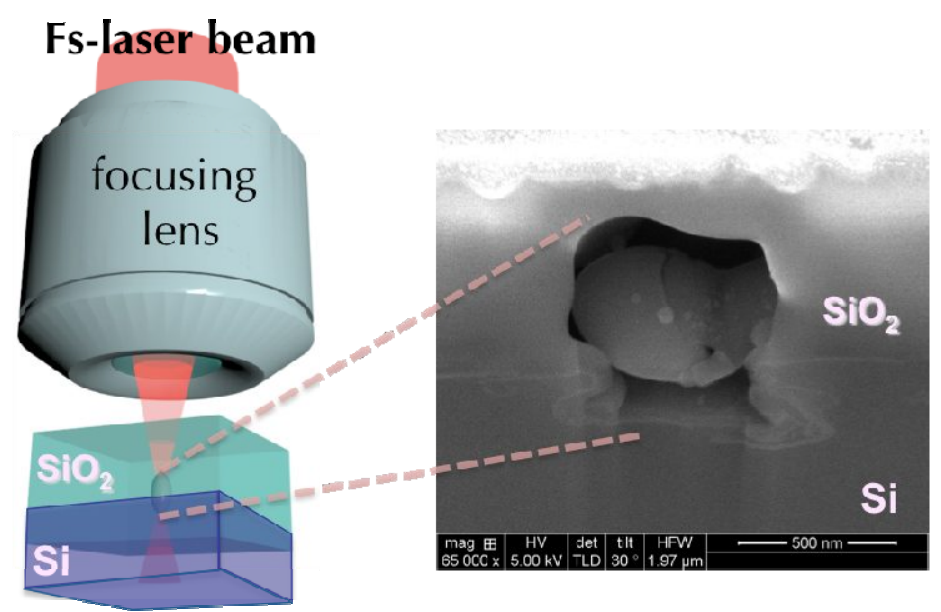

Figure 5. Schematic representation of the laser induced micro-explosion at the $\mathrm{Si} / \mathrm{SiO} 2$ interface with the associated side-view $\mathrm{SEM}$ image of a void produced at the energy of $700 \mathrm{~nJ}$.

The characterization of the laser-affected area, the dense shell surrounding the void and the shock wave affected area by transmission electron microscopy and by Raman micro-spectroscopy will be discuss elsewhere. 


\section{CONCLUSION}

We presented here the structural characterization of a silicon crystal exposed to a strong shock wave induced by femtosecond-laser micro-explosion in confined geometry. We demonstrated the possibility to apply this technique to a non-transparent material by growing a transparent oxide on the sample, thereby it can be applied to virtually any opaque material. Voids at the interface of the silicon and the silica have been observed reveling the creation of high pressure and temperatures in the sample. The studies of tightly focused lasers confined with non-transparent solid encompass a very exciting field for both applied and fundamental science. The presented results demonstrated that it opens up new perspectives for studies of high pressure material at the laboratory tabletop, and unfolds new routes for formation of new material phases expanding the technique possibilities into the domain of non-transparent materials. New mechanism for formation of super-dense materials may lead to understanding of the fundamental properties of Warm Dense Matter.

\section{REFERENCES}

[1] P. F. McMillan, New materials from high-pressure experiments, Nature Materials, 1, 19-25 (2002).

[2] J. M. Léger, J. Haines, M. Schmidt, J.P. Petitet, A.S. Pereira, J.A.H. Da Jordana. Discovery of hardest known oxide. Nature 383, 401 (1996).

[3] A. Salleo, S.T. Taylor, M.C. Martin, W.R. Panero, R. Jeanloz, T. Sands, F.Y. Genin, Laser-driven formation of a high-pressure phase in amorphous silica. Nature Materials 2, 796-800 (2003).

[4] D. G. Hicks, P.M. Celliers, G.W. Collins, J.H. Eggert, S.J Moon, Shock-induced transformation of $\mathrm{Al}_{2} \mathrm{O}_{3}$ and LiF into semiconducting liquids. Phys. Rev. Lett. 91, 035502 (2003).

[5] D. K. Bradley, J.H. Eggert, R.F. Smith, S.T. Prisbrey, D.G.Hicks, D.G. Braun, J. Biener, A.V. Hamza, R.E. Rudd, G.W. Collins, Diamond at 800 GPa. Phys. Rev. Lett. 102, 075503 (2009).

[6] R. Jeanloz, P.M. Celliers, G.W. Collins, J.H. Eggert, K.K.M. Lee, R. Stewart McWilliams, S. Brygoo, P. Loubeyre, High-pressure geoscience special feature: Achieving high-density states through shock-wave loading of precompressed samples. Proc. Nat. Acad. Sci. 104, 9172-9177 (2007).

[7] S. Juodkazis, E. Misawa, E. Gamaly, L. Luther-Davies, B. Hallo, P. Nicolai, V.T. Tikhonchuk, Laser-induced microexplosion inside of sapphire crystal: evidence of multi-megabar pressure, Phys. Rev. Lett. 96, 166101 (2006)

[8] A. Vailionis, E. G. Gamaly, V. Mizeikis, W. Yang, A. Rode, S. Juodkazis, "Evidence of superdense aluminium synthesized by ultrafast microexplosion" Nature Communications 2, 445 (2011).

[9] E. G. Gamaly, A. Vailionis, V. Mizeikis, W. Yang, A. V. Rode, S. Juodkazis, "Warm Dense Matter at the bench-top: Fs-laser-induced confined micro-explosion" High Energy Density Physics, 8, 13-17 (2012).

[10] C. Friedli, N.W. Ashcroft, Aluminum under high pressure. I. Equation of state. Phys. Rev. B 12, 5552 (1975).

[11] J.A. Moriarty, A.K. McMahan, High-pressure structural phase transitions in Na, Mg, and Al. Phys. Rev. Lett. 48, 809 (1982).

[12] J.C. Boettger, S.B. Trickey, High-precision calculation of the equation of state for aluminum. Phys. Rev. B 53, 3007 (1996).

[13] C.J. Pickard, R.J. Needs, Aluminium at terapascal pressures. Nat. Materials 9, $624-627$ (2010).

[14] B. G. Pfrommer, M. Coâté, S. G. Louie, M. L. Cohen, Ab initio study of silicon in the R8 phase, Phys. Rev. B 56, 6662 (1997).

[15] S. Ruffell, K. Sears, A.P. Knights, J.E. Bradby, J. S. Williams, Experimental evidence for semiconducting behavior of Si-XII, Phys. Rev. B 83, 075316 (2011).

[16] J. Crain, G.J. Ackland, J.R. Maclean, R.O. Piltz, P. D. Hatton, G. S. Pawley, Phys. Rev. B 50, 13043 (1994).

[17] J.M. Besson, E. H. Mokhtari, J. Gonzalez, G. Weill, Electrical properties of semimetallic silicon III and semiconductive silicon IV at ambient pressure, Phys. Rev. Lett. 59, 473 (1987).

[18] G. Weill, J. L. Mansot, G. Sagon, C. Carlone, J. M. Besson, Characterisation of Si III and Si IV, metastable forms of silicon at ambient pressure, Semicond. Sci. Technol. 4, 280 (1989).

[19] S. Ruffell, B. Haberl, S. Koenig, J.E. Bradby, J.S. Williams, Annealing of nanoindentation-induced high pressure crystalline phases created in crystalline and amorphous silicon, J. Appl. Phys. 105, 093513 (2009).

[20] E.G. Gamaly, L. Rapp, V. Roppo, S. Juodkazis, A.V. Rode, Generation of high energy density by fs-laser-induced confined microexplosion, New Journal of Physics 15025018 (2013) 